\title{
Observing mesospheric gravity waves with an imaging riometer.
}

\author{
T. Moffat-Griffin ${ }^{\text {a,* }}$, R.E. Hibbins ${ }^{\text {a }}$, K. Nielsen ${ }^{\text {b }}$, M.J. Jarvis ${ }^{\text {a }}$, \\ M.J. Taylor ${ }^{b}$ \\ ${ }^{a}$ British Antarctic Survey, High Cross, Madingley Road, Cambridge, CB3 OET, \\ United Kingdom \\ ${ }^{\mathrm{b}}$ Center for Atmospheric and Space Sciences and Physics Department, Utah State \\ University, Logan, UT, USA
}

\begin{abstract}
Gravity waves play an important role in determining the structure and dynamics of the mesopause region. The Imaging Riometer for Ionospheric Studies (IRIS) at Halley, Antarctica $\left(76^{\circ} \mathrm{S}, 27^{\circ} \mathrm{W}\right)$ is capable of detecting short period mesospheric gravity waves using fluctuations of the ionospheric absorption of cosmic radio noise as a tracer. An analysis technique for quantifying these signatures is presented. The extraction of the wave period, horizontal phase velocity and horizontal wavelength is demonstrated by applying wavelet analysis to synthetic imaging riometer absorption data, which contain known wave features. A mechanism to overcome the limitation on resolvable wavelengths is presented. The effect of noise on the analysis results is also discussed. The application of this technique to extract gravity wave parameters from real imaging riometer data is demonstrated by comparison with those derived from a co-located airglow imager. Extension of this technique will, in future, enable a climatology of year round mesospheric gravity wave properties over Antarctica
\end{abstract}


to be derived. It will also enable further gravity wave climatological studies to be performed using other imaging riometer datasets around the world.

Key words: Imaging riometer, gravity waves, Antarctica, wavelets

\section{$1 \quad 1 \quad$ Introduction}

2

Gravity waves are important for understanding the energy and momentum flow in the mesospause region of Earth, where they can release momentum having propagated upwards from their generation regions in the troposphere and stratosphere. Much of the momentum flux in the mesopause region comes from gravity waves with periods less than 30 minutes (Fritts \& Vincent, 1987). The gravity wave field is not well known in this region and thus the gravity wave parameterisations input into global circulation models (GCMs) will not produce accurate results when compared to real data. The importance of including an accurate representation of the gravity wave field in GCMs is outlined in Fritts et al. (2006). One of the main ways of observing gravity wave activity in the mesopause region is using an airglow imager to infer the amplitude and direction of the short period waves (e.g. Taylor et al. 1995) seen as tracers in the airglow layer at around $87 \mathrm{~km}$ altitude; wintertime climatologies of gravity wave momentum flux over Halley $\left(76^{\circ} \mathrm{S}, 27^{\circ} \mathrm{W}\right)$ and Rothera $\left(68^{\circ} \mathrm{S}\right.$, $68^{\circ} \mathrm{W}$ ) have been generated using airglow imager data (Espy et al., 2004, 2006). However, as the technique requires dark and cloud-free conditions, such imagers are unable to observe during the Antarctic summer. Imaging riometers, on the other hand, are able to observe regardless of these sky conditions and

* Corresponding author.

Email address: tmof@bas.ac.uk (T. Moffat-Griffin). 


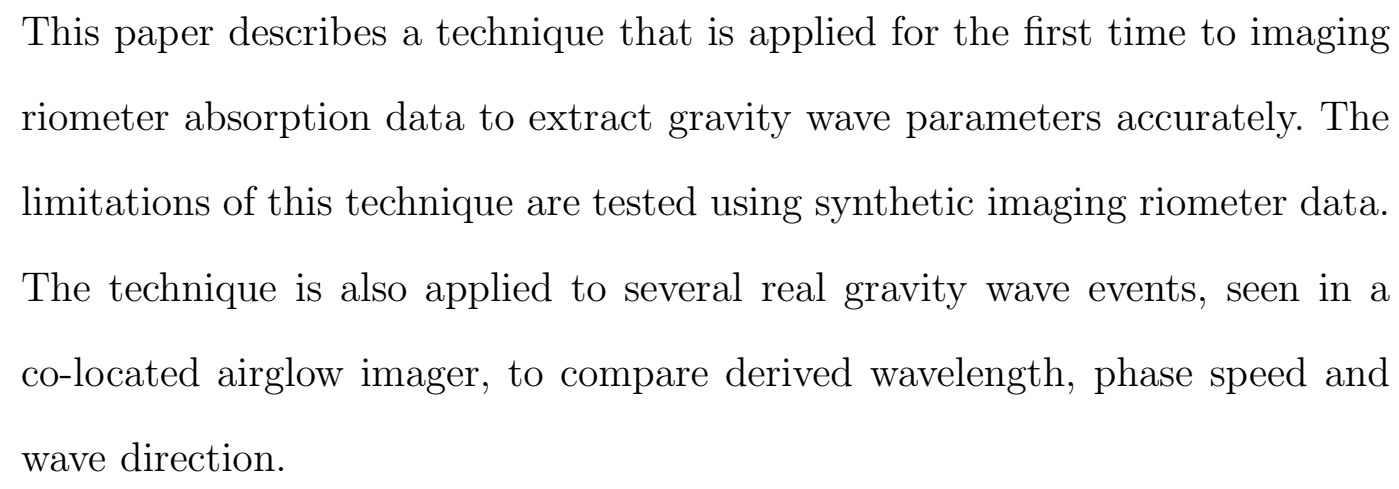

This paper describes a technique that is applied for the first time to imaging riometer absorption data to extract gravity wave parameters accurately. The limitations of this technique are tested using synthetic imaging riometer data. The technique is also applied to several real gravity wave events, seen in a co-located airglow imager, to compare derived wavelength, phase speed and wave direction.

\section{The Imaging Riometer}

A riometer measures the intensity of cosmic radio noise received at the surface of the Earth. If no absorption occurs then the intensity of the cosmic radio noise signal received is cyclic, with the period of a sidereal day, this signal is known as a quiet day curve. If absorption occurs, the intensity of the cosmic radio noise received decreases and hence the absorption in the atmosphere can be determined. Riometer frequencies, usually between 28 and $40 \mathrm{MHz}$, result in a maximum peak in absorption around 90km altitude (Friedrich \& Torkar, 1983) in the D-region of the ionosphere. At Halley, most absorption events are due to auroral sources (Hargreaves \& Jarvis, 1986), although for this study it is required that it is geomagnetically quiet. As the imaging riometer is not being used for usual precipitation studies the peak absorption altitude is likely to differ from $90 \mathrm{~km}$. Work on riometer absorption profiles, (Friedrich \& Torkar, 1983), has shown that as the absorption decreases from $0.5 \mathrm{~dB}$ to $0 \mathrm{~dB}$ the altitude of the peak absorption rises from around $86 \mathrm{~km}$ to around $100 \mathrm{~km}$. The thickness layer of the absorption region has also been shown to vary from $12 \mathrm{~km}$ to $20 \mathrm{~km}$, (Hargreaves, 1980). 
The imaging riometer (Detrick \& Rosenberg, 1990) is an advance on the basic

riometer as it uses a narrow beam antenna array to spatially sample the region of interest in contrast to a spatially isolated measurement from a single wide beam. The imaging riometer for ionospheric studies (IRIS) at Halley has a 64-element crossed dipole array, phased to produce 49 separate beams, each $13^{\circ}$ wide at the $3 \mathrm{~dB}$ power level. A region of over $200 \mathrm{~km}$ by $200 \mathrm{~km}$ at $90 \mathrm{~km}$ altitude is sampled (Rosenberg \& Detrick, 1991). The Halley IRIS operates at $38.2 \mathrm{MHz}$ (a protected band for radio astronomy - so minimising man-made interference). It records data at high (1s) time resolution (all the beams are temporally coincident) but coarse (minimum $22 \mathrm{~km}$ ) spatial resolution (Rose et al., 2000). Figure 1 shows the projection of the riometer beams at $90 \mathrm{~km}$ altitude as defined by the $3 \mathrm{~dB}$ beam projections. The separation between the circular beam projections near the centre is about $22 \mathrm{~km}$; the separation between the non-central elliptical beam projections is significantly larger.

The multi-beam configuration of the Halley IRIS and its field of view make it capable of detecting gravity waves passing horizontally overhead.

\section{Gravity Wave Detection}

Short period gravity waves have been detected in imaging riometer data, from Halley, in a previous study by Jarvis et al. (2003) who demonstrated that a single wave observed in a co-located airglow imager was also present in the central beams of the Halley IRIS. The wave was first seen in the airglow imager at $07: 25 \mathrm{UT}$ on 7 th June 2000 yet did not become apparent in the IRIS data until 08:00UT. Spectral analysis identified 3 wave periods, of which one agreed with the parameters given by the airglow imager. 
Here we explore the possibility of using a more rigorous analysis method that has the potential to be applied semi-automatically to the complete archive (1997 to present day) of imaging riometer data. This provides the potential to build up a year-round climatology of gravity fluxes at Halley, Antarctica.

\subsection{Wavelet Analysis}

Wavelet analysis is a technique that is suited to analysing bursty, frequency varying events that have non-stationary phase throughout the dataset. Gravity waves are such events and thus wavelet analysis lends itself well to their identification.

The Morlet wavelet is used and the transform method outlined in Torrence \& Compo (1998) is implemented in this analysis. The variance of the time series at each wavelet scale (period) is given by the wavelet power spectrum, which is defined as the absolute value of the square of the wavelet transform. The wavelet squared coherency, effectively measuring the cross-correlation between two wavelet power spectra, is used to identify potential wave features that occur at the same period and time range in the two time series. The wavelet coherency phase difference between these coherent features is the key parameter required for IRIS gravity wave parameter extraction and this is provided by the method outlined in Torrence \& Webster (1999).

With knowledge of the distance between the imaging riometer beams projected at a given altitude, the period of the wave feature and its phase difference, the horizontal phase velocity and the horizontal wavelength are calculated.

Equations for the horizontal phase velocity (Equation 1) and the horizontal 
${ }_{92} \quad V_{p}=\frac{2 \pi \Delta x}{\Delta \phi T}$

93 $\quad \lambda=\frac{2 \pi \Delta x}{\Delta \phi}$

where $\Delta x$ is the distance between the beams, $\Delta \phi$ is the phase difference between the beams in radians and $T$ is the wave period in seconds.

The spatial resolution of IRIS puts a limitation on the resolvable horizontal wavelengths of $2 \Delta x$. For the central beams, this results in a lowest resolvable limit of $45 \mathrm{~km}$ for the horizontal wavelength. Any waves that pass through the field of view with horizontal wavelengths shorter than this limit would be difficult to accurately resolve and any parameters derived from them would therefore not be reliable. A method to eliminate this problem has been developed and requires a wave feature to be detected in three linearly adjacent beams over the same period and time range. Figure 2 illustrates the separation between three of the beams and using simple wave theory, Equation 3 is derived.

$\frac{d_{a b}}{d_{b b 2}}=\frac{\Delta \phi_{a b}}{\Delta \phi_{b b 2}}$

The beam separations $d_{a b}$ and $d_{b b 2}$ are known, and the phase difference ratio, on the right-hand side of Equation 3, can be adjusted using $\pm 2 n \pi$ until the best match to the beam separation ratio is found, where $n$ is any integer. The adjusted phase difference values then represent the actual phase difference. This adjustment can then be applied to the phase differences in Equations 1 and 2 to calculate the true wavelength and phase velocity of the wave feature. The ability to calculate this necessary adjustment relies on the fact that the 
beam separations at any fixed altitude are neither equal nor have an integer ratio.

Finally, the direction of the wave can be calculated by using a technique outlined in Donelan et al. (1996) for application to ocean waves. Two phase differences of a wave feature, measured in near orthogonal directions, are required. For the Halley case, the central beam phase differences between the IRIS north-south and east-west beams are at exactly $90^{\circ}$ and the wave direction is given by Equation 4:

$$
\theta=\operatorname{atan}\left(\frac{\Delta \phi_{a c}}{\Delta \phi_{a b}}\right)
$$

Where the subscripts $a c$ refer to the north-south pair of beams and $a b$ the east-west pair of beams. For the case here $0^{\circ}$ and $360^{\circ}$ are pointing north, with the angles increasing in a clockwise direction.

\section{Synthetic Data Results}

\subsection{Attenuation of the IRIS beams}

The beam profile of each of the central IRIS beams approximates a Gaussian, where the beam width is taken to be the FWHM. This shape results in the attenuation of the signal observed. The beam widths for beams $a, b$ and $b 2$ are, at $90 \mathrm{~km}$ altitude, $17.6 \mathrm{~km}, 17.9 \mathrm{~km}$ and $18.8 \mathrm{~km}$ respectively. Figure 3 shows how the amplitude of different wavelength horizontal waves would be attenuated by each beam. It shows that the three beams exhibit a similar attenuation pattern and that waves with a horizontal wavelength of $10 \mathrm{~km}$ and less have 
their amplitudes reduced by a factor of 100,000, making them undetectable.

There is no observed phase shift associated with this attenuation.

The results in the next sections are for synthetic waves, already attenuated by the beams, and discuss the effect of signal noise on the detection capabilities.

\subsection{Extracting wave parameters}

Time series for the five beams in Figure 1 are produced for unit amplitude waves with various wavelengths, frequencies and directions. The waves are of the form of Equation 5 .

$$
A(x, y, t)=\sin \left(k_{x} x+k_{y} y-\omega t\right)
$$

where $A$ is the wave amplitude, $k_{x}$ and $k_{y}$ are the $\mathrm{x}$ and $\mathrm{y}$ components of the wavenumber, $\omega=\frac{2 \pi}{T}$ and $t$ is the time in seconds. Figure 4 shows a typical example of the different beam signatures of a synthetic wave. In this instance the wave has horizontal wavelength of $20 \mathrm{~km}$, a speed of $19 \mathrm{~ms}^{-1}$ and a direction of $70^{\circ}$.

The wavelet coherency spectrum of each of the five pairs of beams was generated and the phase differences from the regions of highest coherency were determined. These were then adjusted, as shown in Section 3.1, and the horizontal wavelength, horizontal phase velocity and wave direction were calculated. Table 1 shows the success of the technique with various wavelengths and directions keeping the period constant at 1057s. This illustrates that it is possible to detect short horizontal wavelengths down to $15 \mathrm{~km}$ wavelength reliably within the geometry of the 5 central beams. 
This illustrates that the technique works for situations where the signal (wave) amplitude is large compared to the noise amplitude (a signal to noise amplitude ratio of around 10) and the wavelength is $15 \mathrm{~km}$ or longer. As real IRIS data is noisy, compared to the amplitude of the gravity wave signal, (Jarvis et al., 2003), the next section tests the limits of the technique by increasing the noise level (decreasing the signal to noise ratio).

\subsection{Noisy data}

The same time series as above were reproduced but with different signal to noise ratios. The effect of increasing the noise in the time series is shown by taking the standard deviation of the difference between the predicted and actual wave parameter and comparing it to the log of the signal to noise ratio. Figure 5 shows this type of plot for the standard deviations of the horizontal phase velocity and direction differences.

As the noise amplitude increases, the standard deviation of the parameter difference increases. The error of the predicted results increases. In both plots in Figure 5 a sharp increase in standard deviation can be seen when the signal to noise ratio is around 0.3. Beyond this point, the parameter predicted will become more unreliable.

To determine the extent to which the real IRIS data can be analysed in this manner a test is currently being developed that allows an estimate of the signal to noise amplitude ratio to be obtained. However, in the meantime, a simple estimation of the noise amplitude in real IRIS data can be performed by filtering out any period longer than 3 minutes and taking the standard 


\section{Real IRIS Data}

A test of the technique has been performed by comparing IRIS data to seven gravity wave events observed in the $\mathrm{OH}$ airglow imager. These events have been chosen from a climatological study that was done using the airglow imager, (Nielsen , 2007). This study revealed a large number of extensive gravity waves measured in the $\mathrm{OH}$ emission over Halley. The wave parameters spanned from $10-60 \mathrm{~km}$ in horizontal wavelength, $5-100^{-1}$ in observed phase speed, and 5-30 minutes in observed period, with typical values of $26 \mathrm{~km}, 48 \mathrm{~ms}^{-1}$, and 10 minutes, respectively. For this test seven waves exhibiting wavelengths larger than $15 \mathrm{~km}$ and periods greater than 10 minutes were selected for comparison. These criteria are based on the observing capabilities of the IRIS. Table 2 shows the dates and times of the events used in this study, plus the $\mathrm{K}_{p}$ index. The wavelet analysis technique outlined above is applied to each IRIS dataset for the relevant date and time.

The events were analysed and strong wave features, coincident in period and time, were observed in their wavelet coherency spectra. The wavelet coherency spectra for the East-West beam pairs of event E are shown in Figures 6 and 7 with the wave features highlighted. Figure 8 shows the regions where all four beam pairs have a wavelet coherency greater than 0.5 for event $\mathrm{E}$ and the horizontal solid line marks the observed period seen in the airglow imager. This enables a clearer identification of the wave event than Figures 6 and 7. Figure 9 is the same plot type as Figure 8 but for all the other events studied in this paper. It shows that the wave events consistently seen in all 
5 central beams of the IRIS data have similar periods to those observed in the airglow imager data. From the coherency spectra, the phase differences between the features in different beams were estimated, and their horizontal wavelengths, phase velocities and directions were calculated. Figures 10, 11, 12 and 13 compare the horizontal phase velocity, period, horizontal wavelength and direction determined by the $\mathrm{OH}$ airglow imager and IRIS for each gravity wave event. Figures 10, 11 and 12 show that there is agreement, including errors, between the velocities, period and wavelengths of waves observed by both instruments. Event D is the exception to this, where the predicted wave velocity by IRIS is about $60 \%$ of the value derived from the airglow imager data. Table 2 shows that for this event the $\mathrm{K}_{p}$ is 3 , the highest of any event studied. This level of geomagnetic activity could be sufficient to increase the variability in the absorption level, obscuring the gravity wave signal more than a $\mathrm{K}_{p}$ level of 2 or less would. The small differences in values could in part be because the instruments are looking at slightly different altitudes. For the direction comparisons shown in Figure 13 it is evident that the IRIS and airglow imager direction results do not agree for all the events. There have not been any signal to noise estimates made for the IRIS data used in this paper but some of the results imply that the noise levels may be high enough to cause reliability issues with the direction measurements. A technique to estimate the signal to noise level in the IRIS data is under development.

\section{Summary}

Studies with synthetic gravity wave IRIS data and real IRIS data have shown that the wavelet analysis technique outlined in this paper is successful, within 
its limits, in extracting gravity wave parameter information. The use of synthetic data have provided a set of limits on the spectrum of gravity waves that can be detected, and the accuracy of these predictions due to noise. Waves with a horizontal wavelength less than $15 \mathrm{~km}$ are difficult to obtain parameters for and parameters derived from a time series where the signal to noise ratio is less than 0.25 have a much larger uncertainty associated with them than those with a higher signal to noise ratio.

The results in Section 5 are encouraging. Comparisons between the airglow imager results and the IRIS results demonstrate that the IRIS is capable of detecting gravity waves and that the wave period, wavelength, velocity and direction can be successfully extracted using a wavelet analysis technique. The noise levels in the IRIS data affect the reliability of the direction results; so being able to determine accurately the signal to noise level of the IRIS data would increase the confidence in any future studies where the IRIS is used in a stand-alone situation to detect gravity waves in daylight.

With a co-located airglow imager it is easy to verify that the waves observed in IRIS are in the D/E-region not the F-region; however in the summertime this comparison technique cannot be used. Kressman (1976) shows that when foF2 is below $5 \mathrm{MHz}$, the absorption due to the F-region is less than $0.1 \mathrm{~dB}$. Thus, a F-region gravity wave perturbation could cause a variation in the absorption of around $0.01 \mathrm{~dB}$, which would be detectable. The use of an ionosonde, colocated with the IRIS, is a possible method of identifying F-region gravity waves, (Kressman , 1976). Ionosonde data could be used in conjunction with the stand-alone IRIS data to determine whether waves seen in the IRIS are occuring in the F-region - if a wave of a certain period is seen in the IRIS data but not the ionosonde then it is likely to be a wave in the mesosphere. 
The analysis technique outlined in this paper will be applied to the entire Halley IRIS dataset to build up a climatology of mesospheric gravity wave characteristics over this region. However the technique can be applied to any 49 or more beam imaging riometer. There are many imaging riometers situated across the polar regions. The potential to exploit these additional datasets and generate many polar climatologies is huge and would contribute to our understanding of the mesospheric gravity wave field over the polar regions greatly.

\section{Acknowledgements}

The Imaging Riometer for Ionospheric Studies (IRIS) is jointly supported by the Natural Environment Research Council and by the U.S. office of polar programmes grant OPP-0003881. Thanks go to the Halley field engineers and Cambridge staff who deployed the IRIS system and help to maintain the instrument and its data.

\section{References}

Detrick, D.L.\& Rosenberg, T.J., A phased-array radiowave imager for studies of cosmic noise absorption, Radio Sci., 25, 325-338, 1990

Donelan, M.A., Drennan, W.M. \& Magnusson, A.K., Nonstationary analysis of the directional properties of propagating waves, J. Phys. Oceanogr., 26, 1901-1914, 1996

Espy, P.J., Jones, G.O.L., Swenson, G.R., Tang, J \& Taylor, M.J., Seasonal 
variations of the gravity wave momentum flux in the Antarctic mesosphere and lower thermosphere, J. Geophys. Res., 109, D23109, 2004

Espy, P.J., Hibbins, R.E., Swenson, G.R., Tang, J., Taylor, M.J., Riggin, D.M. \& Fritts, D.C., Regional variations of mesospheric gravity-wave momentum flux over Antarctica, Ann. Geophys., 24, 81-88, 2006

Friedrich, M. \& Tokar, K.M., High-latitude plasma densities and their relation to riometer absorption, J. Atmos. Terr. Phys., 45, 127-135, 1983

Fritts, D.C. \& Vincent, R.A., Mesospheric momentum flux studies at Adelaide, Australia, J. Atmos. Terr. Phys., 44, 605-619, 1987

Fritts, D.C., Vadas, S.L., Wan, K. \& Werne, J.A., Mean and variable forcing of the middle atmosphere by gravity waves, J. Atmos. Sol. Terr. Phys., 68, 247-265, 2006

Hargreaves, J.K., D-region electron densities observed by incoherent-scatter radar during auroral-absorption spike events, J. Atmos. Terr. Phys, 42, $783-789,1980$

Hargreaves, J.K. \& Jarvis, M.J., The multiple riometer system at Halley, Antarctica, Surv. Bull., 72, 13-23, 1986

Jarvis, M.J., Hibbins, R.E., Taylor, M.J. \& Rosenberg, T.J., Utilizing riometery to observe gravity waves in the sunlit mesosphere, Geophys. Res. Lett., 30,2003

Kressman, R.I., Riometer studies at South Georgia, Br. Antact. Surv. Bull., 46, 15-23, 1976

Nielsen, K., Climatology and Case Studies of Mesospheric Gravity Waves Observed at Polar Latitudes, PhD dissertation, Utah State University, 2007

Rose, M.C., Jarvis, M.J, Clilverd, M.A, Maxfield, D.J. \& Rosenberg, T.J., The effect on snow accumulation on imaging riometer performance, Radio Sci., $35,1143-1153,2000$ 
Rosenberg, T.J. \& Detrick, D.L., A comparative study of imaging and broadbeam riometer measurements: The effect of spatial structure on the frequency dependence of auroral absorption, J. Geophys. Res., 96, 1779317803, 1991

Taylor, M.J., Bishop, M.B. \& Taylor, V., All-sky measurements of short period waves imaged in the $\mathrm{OI}(557.7 \mathrm{~nm}), \mathrm{Na}(589.2 \mathrm{~nm})$ and near infrared $\mathrm{OH}$ and O2(0,1) nightglow emissions during the ALOHA-93 campaign, Geophys. Res. Lett., 22, 2833, 1995

Torrence, C. \& Compo, G.P., A practical guide to wavelet analysis, Bull. Amer. Met. Soc., 79, 61-78, 1998

Torrence, C. \& Webster, P.J., Interdecadal changes in the ENSO-monsoon system, J. Climate, 12, 2679-2690, 1999

Table 1: Original and predicted wavelengths $(\mathrm{km})$, phase velocities $\left(\mathrm{ms}^{-1}\right)$ and directions $\left(\theta^{\circ}\right)$ for synthetic data inputs. The need for adjustment is also indicated. At a horizontal wavelength of $10 \mathrm{~km}$ the technique is seen to break down.

Table 2: Dates and times of the gravity wave events seen in the $\mathrm{OH}$ airglow imager and studied in this paper. The $\mathrm{K}_{p}$ index is included to show the level of geomagnetic activity. Those times with an asterisk are where the entire wave event duration could not be determined due to auroral events obscuring part of the field of view.

Figure 1: Projection of the imaging riometer beams onto a horizontal plane at $90 \mathrm{~km}$ altitude, as defined by their $3 \mathrm{~dB}$ contour. The five westward and northward beams discussed in the text are shaded and labelled. 
Figure 2: Diagram of beam separations and the phase differences between beam pairs.

Figure 3: The effect on the observed wavelength of the different beam widths for different wavelength waves. The solid line is for beam 'a', the dotted line is for beam 'b' and the dashed line is for beam 'b2'.

Figure 4: A wave of wavelength $20 \mathrm{~km}$, velocity $19 \mathrm{~ms}^{-1}$, direction of $70^{\circ}$ and a period 1057 seconds with a signal to noise ratio of 10 , as seen by the 5 beams in figure 1.

Figure 5: The top plot shows the standard deviation of the direction difference versus $\log ($ signal/noise $)$, the bottom plot shows the standard deviation of the velocity difference versus $\log ($ signal/noise).

Figure 6: Wavelet coherency plot between beams a and b for event E. The pink line highlights the wave feature used to derive the wave parameters. The thick black contour lines indicate a coherency greater than 0.5 (where the maximum is 1). The additional features seen in the figure do not fulfil the criteria of being seen in additional beams.

Figure 7: As for Figure 6 but for beams b and b2

Figure 8: Combined coherency plot for all four beam pairs for event E. The shaded regions are where the coherency is greater then 0.5 in all four beam pairs. The solid red line indicates the period of the wave seen in the airglow imager.

Figure 9: Combined coherency plots for the remaining events studied in this paper. The shaded regions are where the coherency is greater then 0.5 in all 
four beam pairs. The solid line indicates the period of the wave seen in the airglow imager.

Figure 10: Comparison of derived horizontal phase velocities of each gravity wave event outlined in Table 2. The squares represent the $\mathrm{OH}$ imager results with associated errors; the triangles represent the IRIS results with associated results.

Figure 11: As for Figure 10 but for wave period.

Figure 12: As for Figure 10 but for horizontal wavelength

Figure 13: As for Figure 10 but for wave direction.

Table 1

\begin{tabular}{lcccccc}
\hline orig $\lambda$ & orig $\mathrm{V}_{p}$ & orig $\theta^{\circ}$ & predicted $\lambda$ & predicted $\mathrm{V}_{p}$ & predicted $\theta^{\circ}$ & adjust \\
\hline 60.0 & 56.7 & 70.0 & 59.5 & 56.3 & 69.7 & no \\
50.0 & 47.6 & 120.0 & 50.3 & 47.6 & 120.2 & no \\
40.0 & 37.8 & 200.0 & 39.6 & 37.5 & 198.6 & yes \\
30.0 & 28.3 & 280.0 & 29.3 & 27.8 & 280.2 & yes \\
20.0 & 19.6 & 320.0 & 20.7 & 19.6 & 318.7 & yes \\
15.0 & 14.3 & 330.0 & 15.5 & 14.1 & 328.5 & yes \\
10.0 & 9.4 & 340.0 & 18.1 & 17.2 & 221.1 & yes \\
\hline
\end{tabular}


Table 2

\begin{tabular}{llll}
\hline & & & \\
Event & Day of year (2000) & Time of event & $\mathrm{K}_{p}$ \\
\hline A & 089 & $01: 30-03: 30$ & 0.5 \\
B & 100 & $01: 00^{*}$ & 2.0 \\
C & 119 & & \\
& & $22: 00-00: 00$ & 2.0 \\
D & 122 & $23: 00^{*}$ & 3.0 \\
E & 125 & & \\
F & 154 & $04: 30-07: 00$ & 1.0 \\
G & 187 & $22: 30-00: 00$ & 2.0 \\
& & & \\
\hline
\end{tabular}




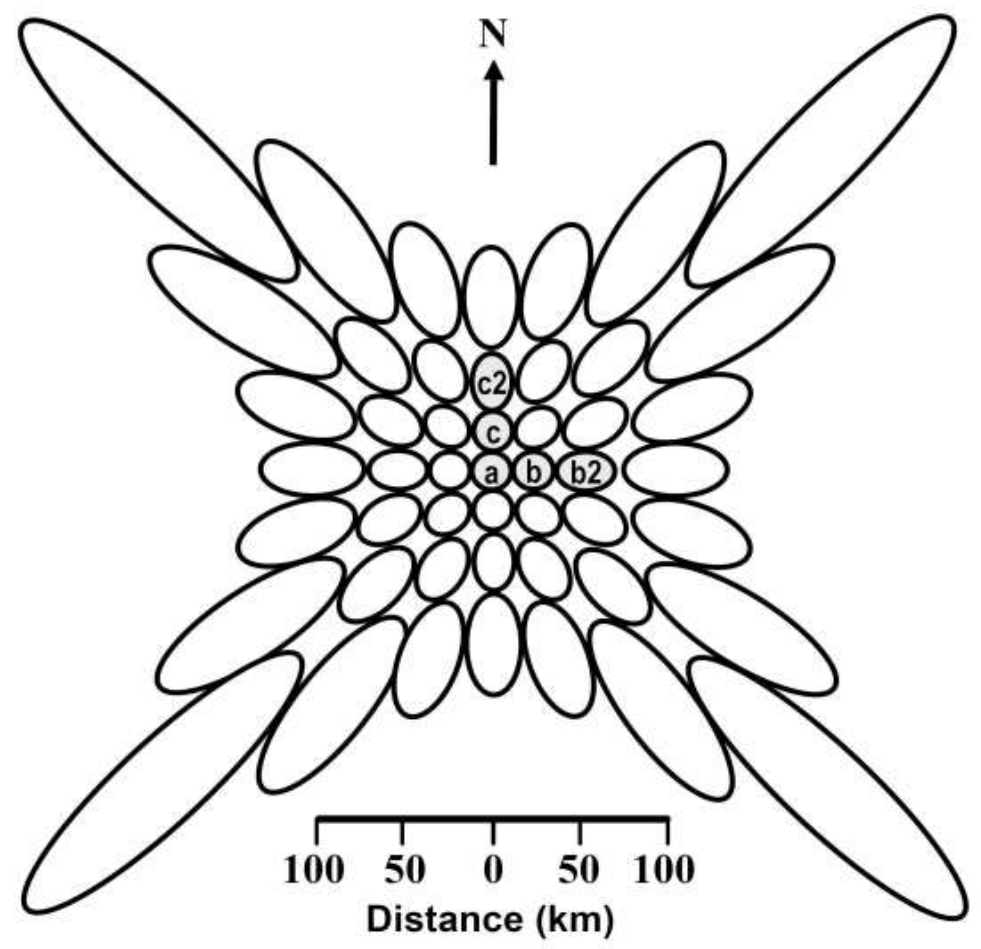

Fig. 1.

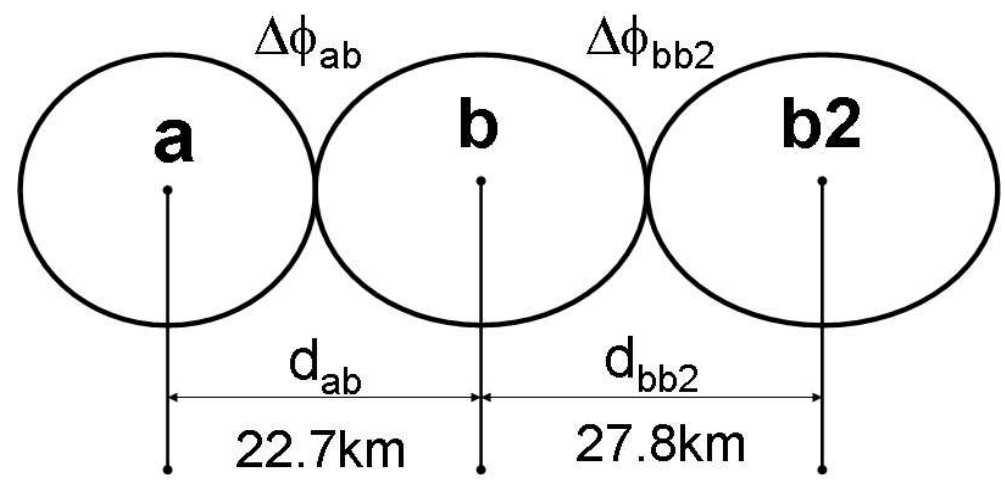

Fig. 2. 


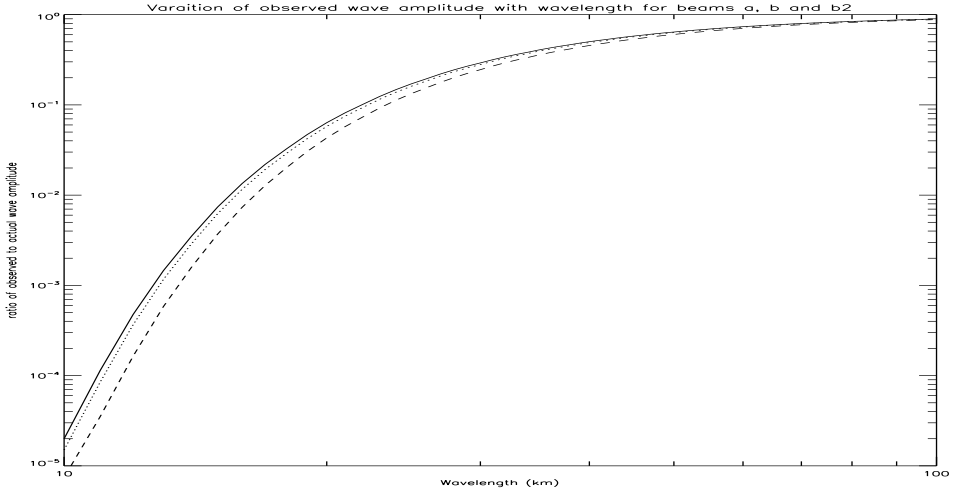

Fig. 3.

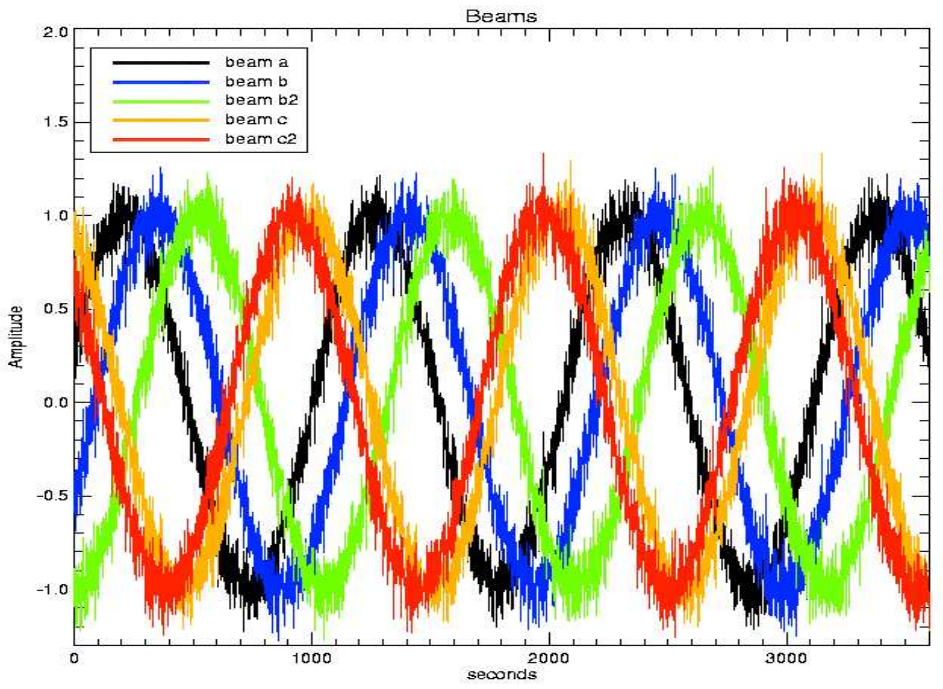

Fig. 4. 

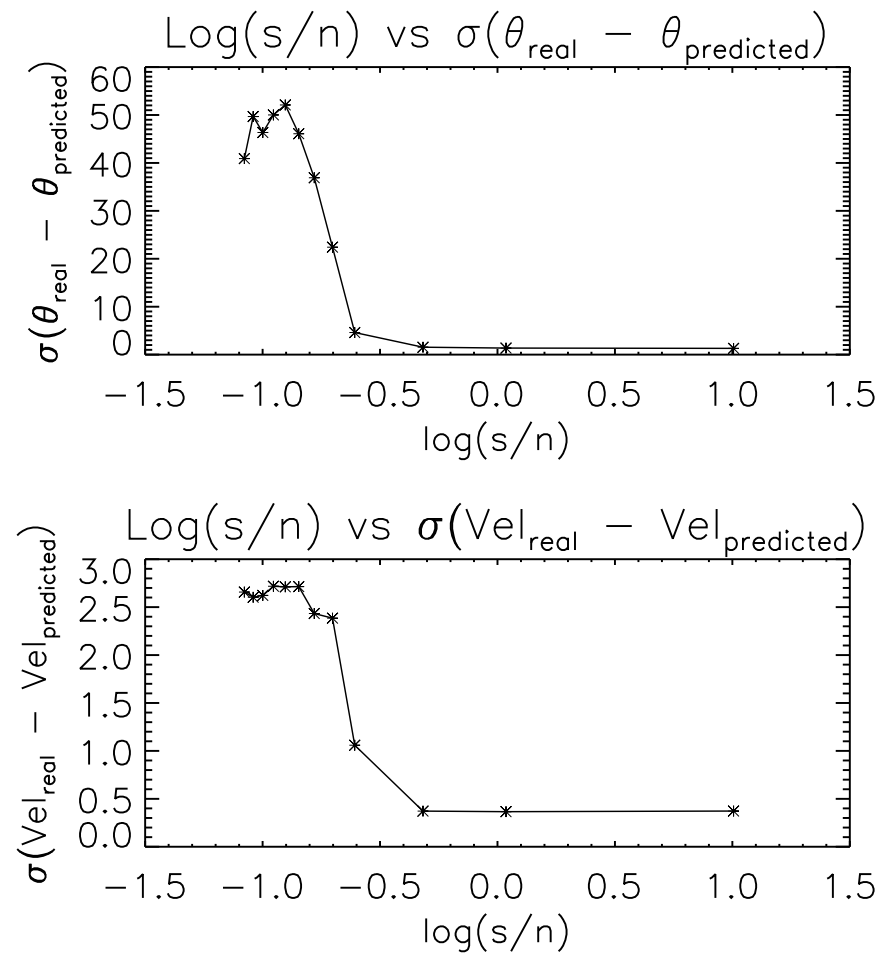

Fig. 5.

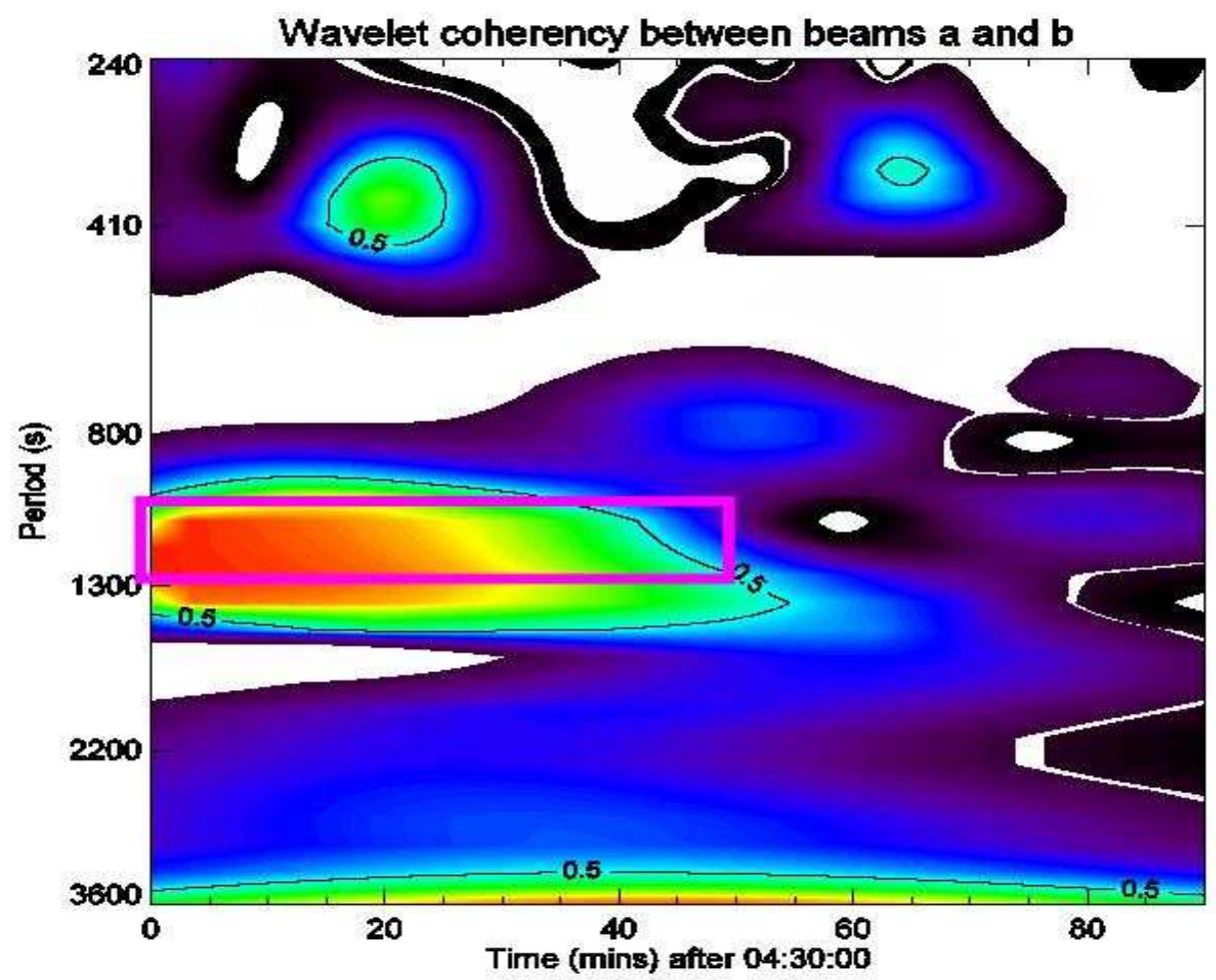

Fig. 6. 


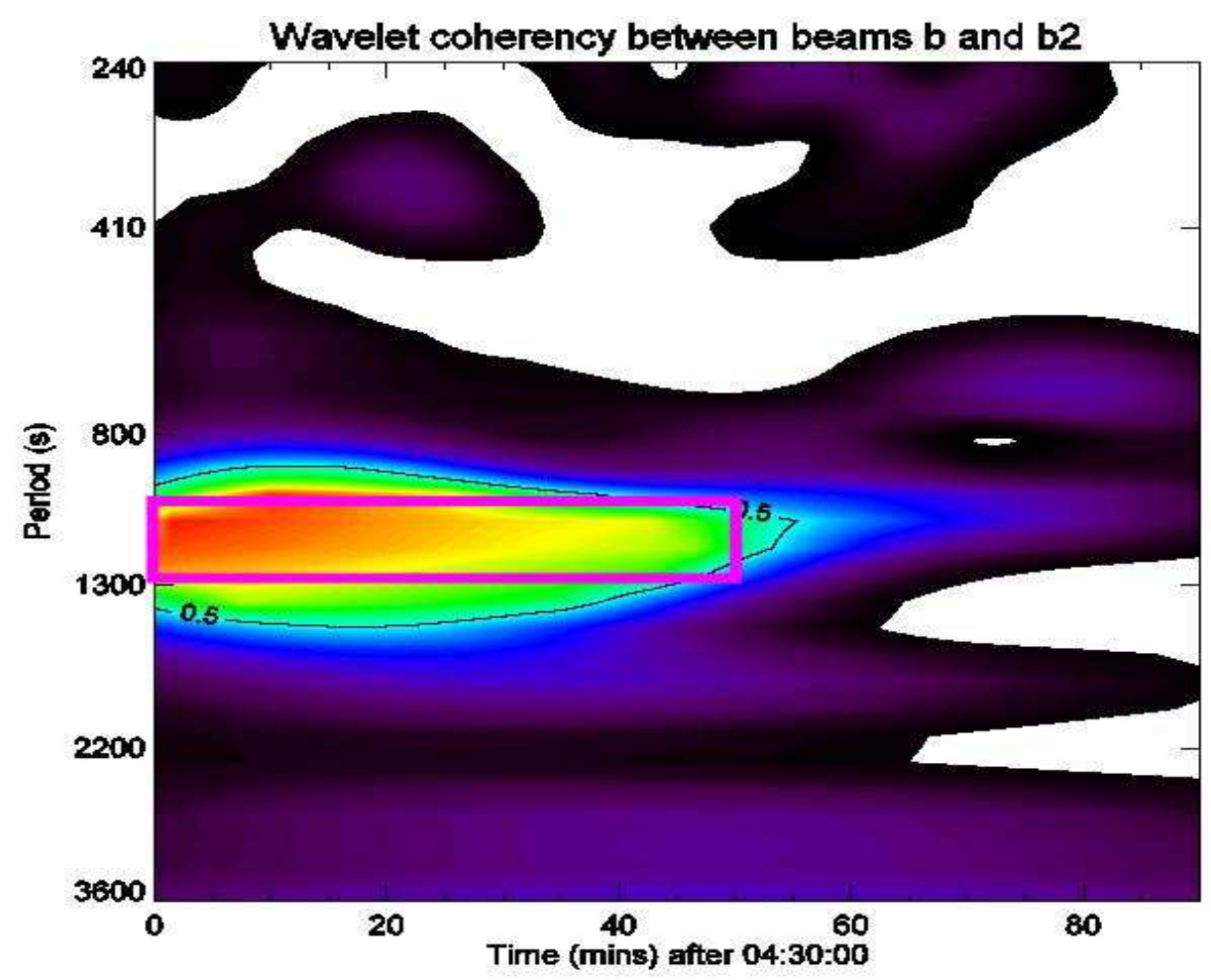

Fig. 7.

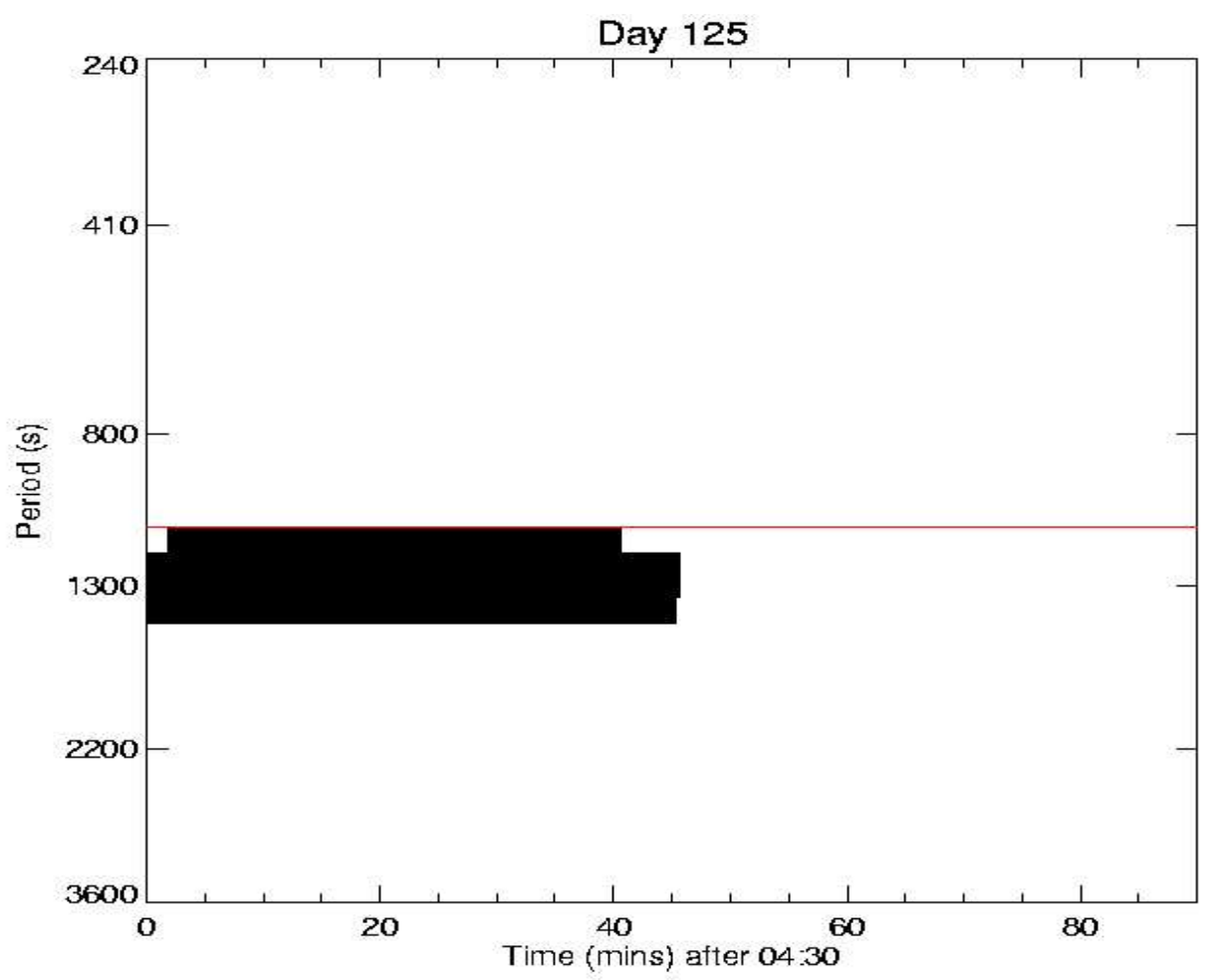

Fig. 8. 

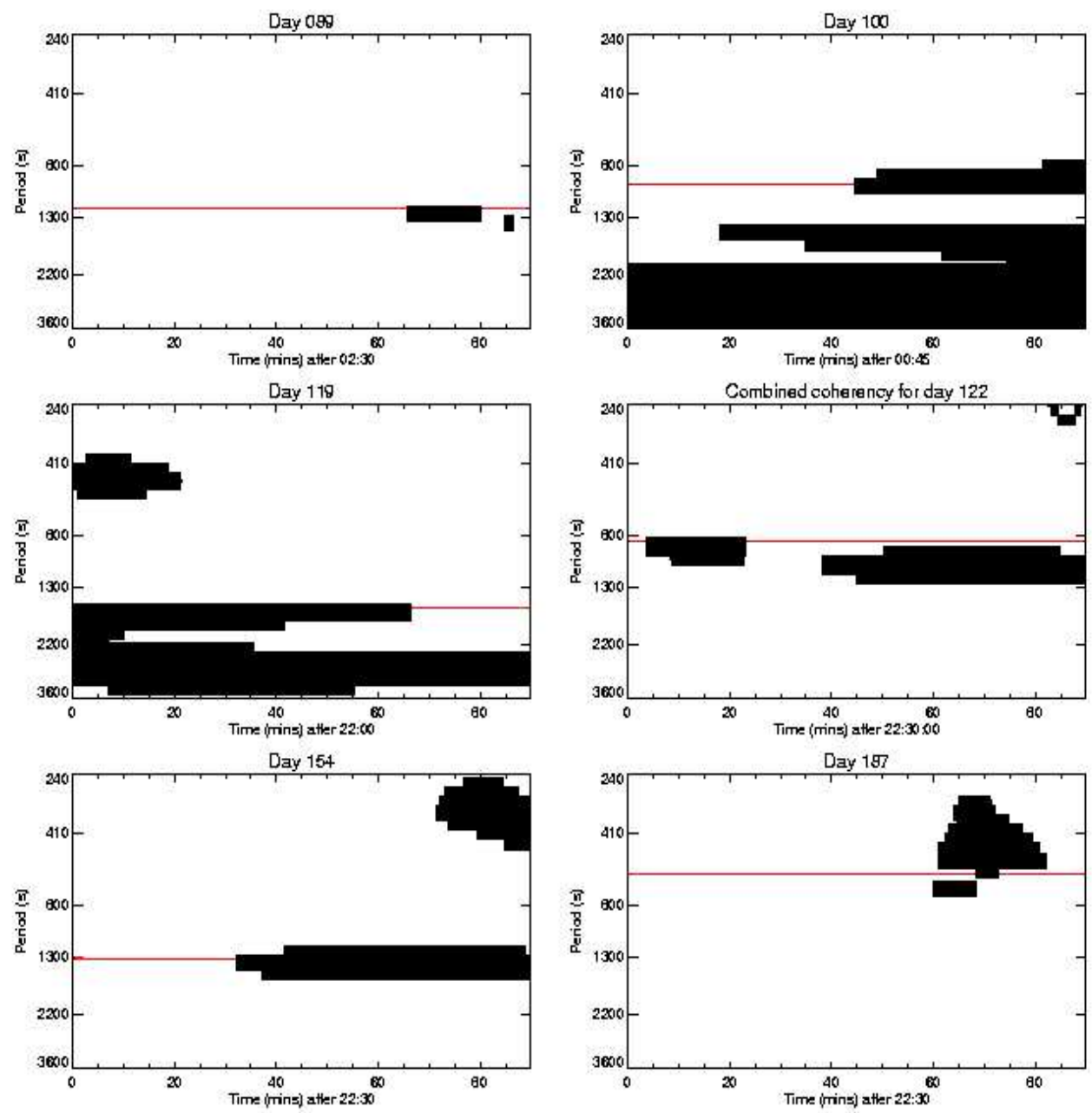

Fig. 9. 
Comparison of $\mathrm{OH}$ imager and IRIS gravity wave horizontal phase velocities

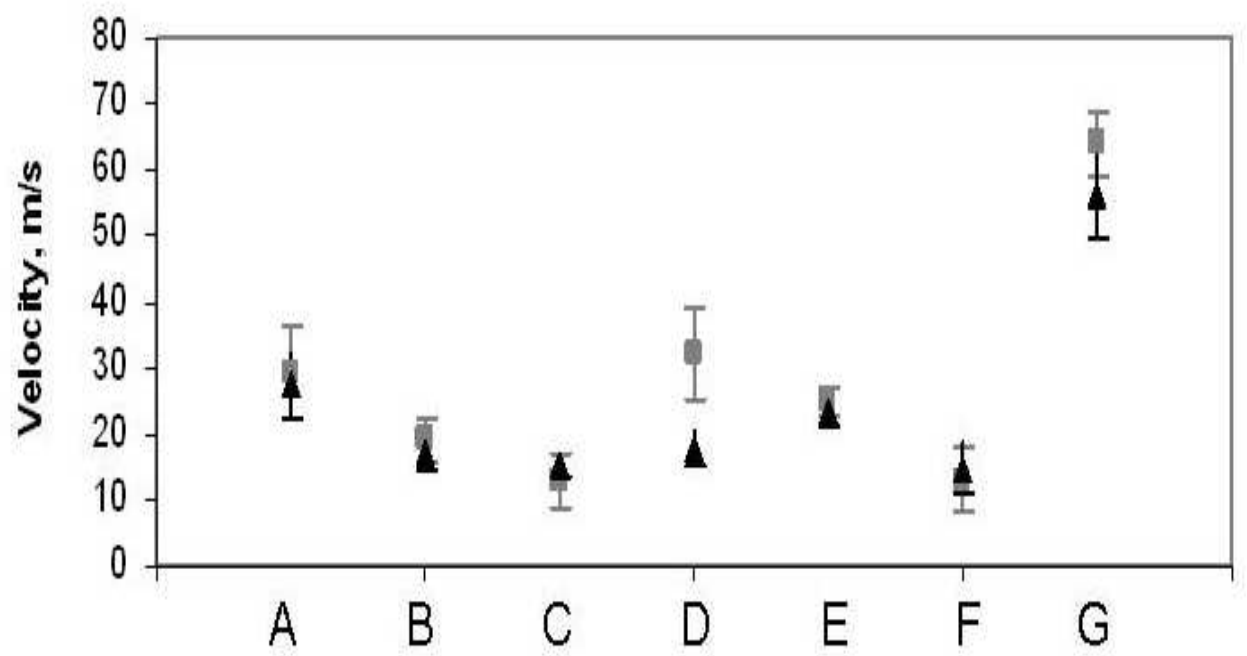

Fig. 10.

Comparison of OH imager and IRIS gravity wave periods

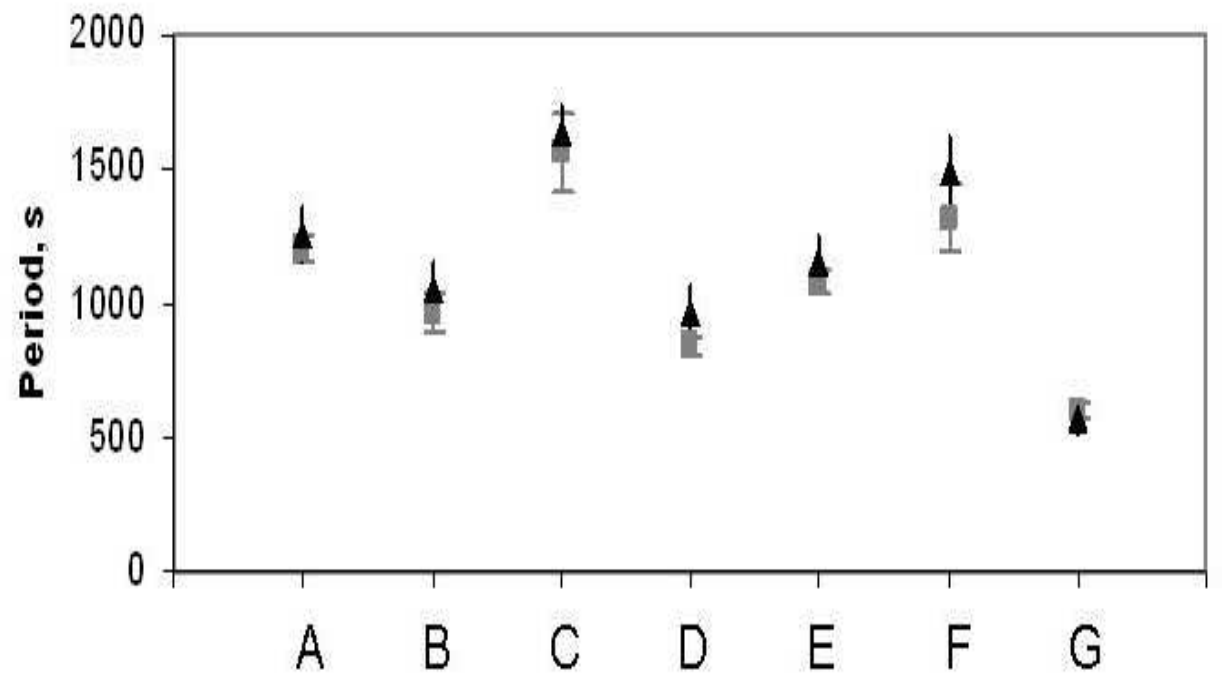

Fig. 11. 


\section{Comparison of $\mathrm{OH}$ imager and IRIS gravity wave horizontal wavelength}

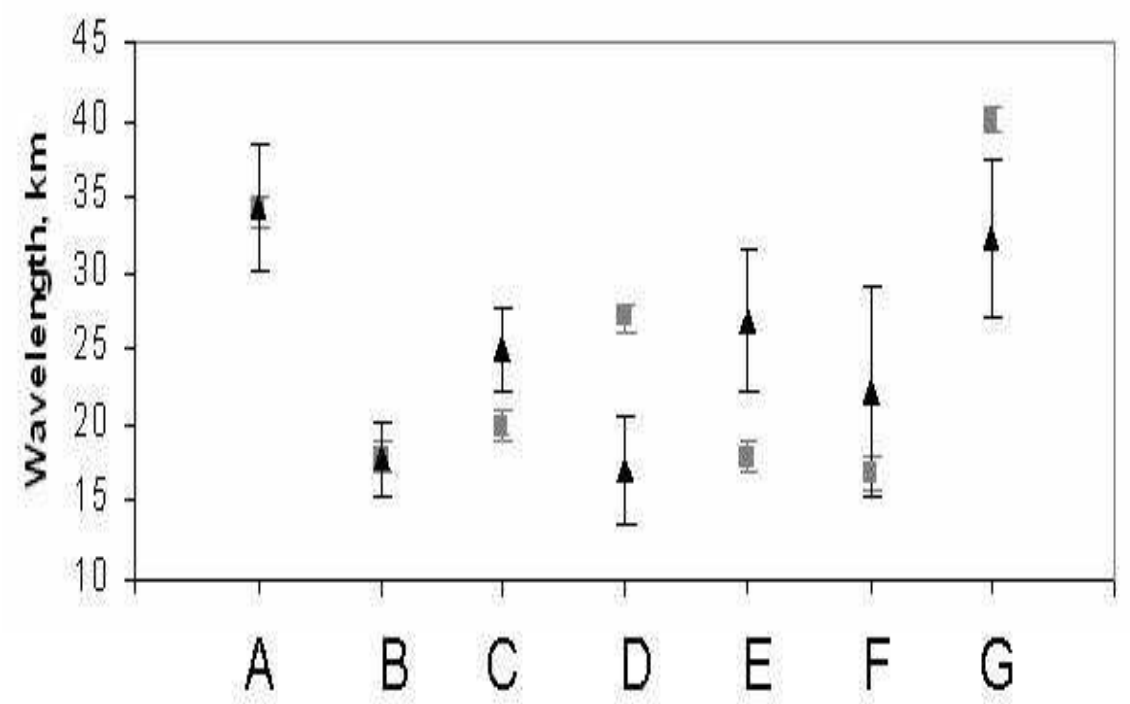

Fig. 12 .

Comparison of $\mathrm{OH}$ imager and IRIS gravity wave direction

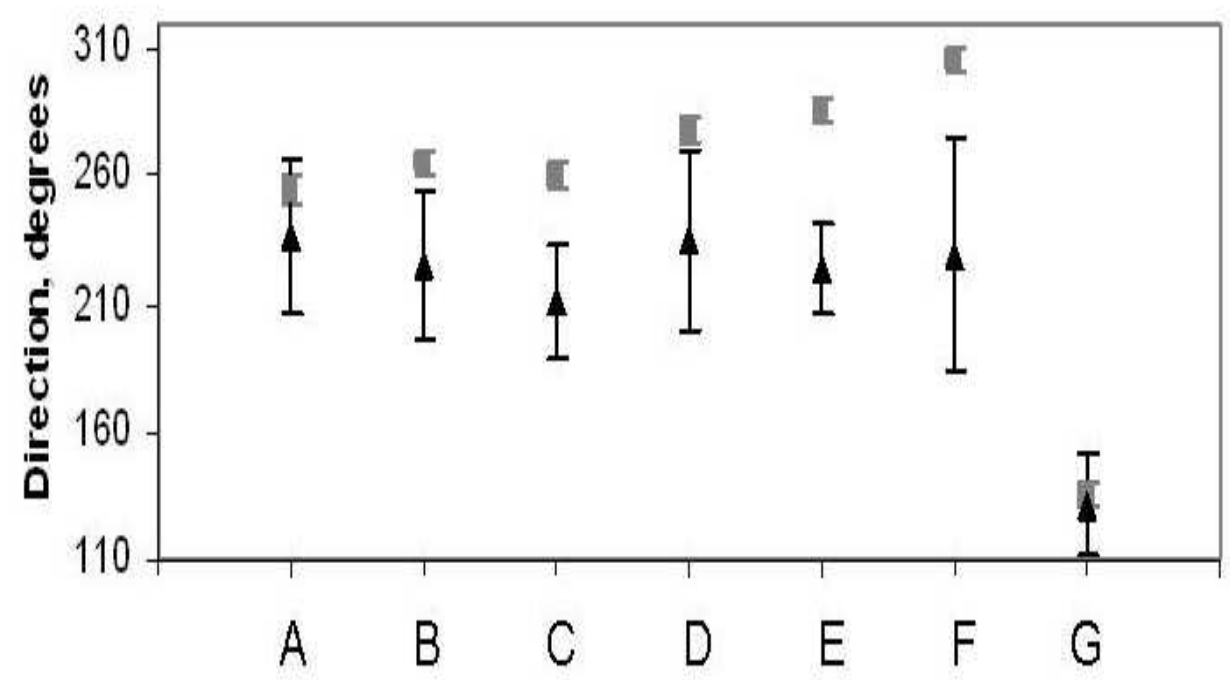

Fig. 13. 